\title{
Valor pronóstico de progresión de las moléculas reguladoras del ciclo celular en tumores vesicales T1G3
}

\author{
J.A. Queipo Zaragozá, J.L. Ruiz Cerdá, L. Palmero Martí, L.A. Rubio Martínez*, \\ F. Vera Sempere*, J.F. Jiménez Cruz
}

Servicio de Urología, Servicio de Anatomía Patológica*. Hospital Universitario La Fe. Valencia.

Actas Urol Esp 2005; 29 (3): 261-268

\section{RESUMEN \\ VALOR PRONÓSTICO DE PROGRESIÓN DE LAS MOLÉCULAS REGULADORAS DEL CICLO CELULAR EN TUMORES VESICALES T1G3}

Introducción y Objetivos: El tumor vesical T1G3 constituye el grupo de tumores superficiales más agresivo. Nuevos factores pronósticos en el campo de la citogenética y la biología molecular han sido analizados, con resultados a menudo contradictorios, siendo escasos los trabajos específicos en tumores T1G3. Nuestro objetivo es determinar si en este grupo de tumores los marcadores inmunohistoquímicos presentan valor predictivo de progresión clínicamente útil, y por tanto con validez para indicar una actitud terapéutica precoz más idónea.

Material y Métodos: Estudio retrospectivo de una serie de 83 pacientes afectos de tumor vesical T1G3, sobre los que analizamos un total de 14 variables; entre los nuevos factores predictivos: la determinación inmunohistoquímica de las proteínas reguladoras del ciclo celular: p53, p21 y bcl-2, así como la proteína Ki-67 como marcador de proliferación celular. Mediante análisis de regresión logística establecemos las variables pronósticas independientes para progresión tumoral.

Resultados: El punto de corte establecido para Ki67 y p53 fue el 40\% de células inmunomarcadas, $20 \%$ para p21 y $10 \%$ para Bcl-2. El análisis univariante puso de manifiesto diferentes tasas de progresión y tiempos libres de progresión en función de la inmunotinción de Ki67 y p53; sin embargo, la regresión logística demostró que solo la inmunohistoquímica de p53 presentaba valor predictivo independiente.

Conclusiones: La determinación inmunohistoquímica de p53 presenta valor predictivo de progresión clínicamente útil en tumores vesicales T1G3, de manera que su determinación puede constituir un factor fundamental en las estrategias de tratamiento de estos tumores.

Palabras clave: T1G3. Inmunohistoquímica. p53.

\section{ABSTRACT \\ PROGNOSTIC VALUE FOR PROGRESSION OF THE REGULATING PROTEINS OF THE CELLULAR CYCLE IN PT1G3 BLADDER TUMOURS}

Introduction and objective: Bladder tumor T1G3 constitutes the group of superficial tumors more aggressive. New prognostic factors in the field of the cytogenetics and molecular biology have been analyzed, with often contradictory results, being little the specific works in tumors T1G3. Our objective is to determine if in this group of tumors the immunohistochemical markers present predictive value of clinically useful progression, and therefore with validity to indicate more suitable a precocious therapeutic attitude

Material and methods: Retrospective study of a series of 83 patients affected of bladder tumor T1G3, on which we analyzed a total of 14 variables; between the new predictive factors: the immunohistochemical determination of regulating proteins of the cellular cycle: p53, p21 and bcl-2, as well as the Ki-67 protein like marker of cellular proliferation. By means of logistic regression analisys we establish the independent prognostic variables for tumorlike progression.

Results: The cut point established for Ki67 and p53 was 40\% of inmmunomarked cells, 20\% for p21 and 10\% for Bcl-2. The univariant analysis showed different rates from progression and free times of progression based on the immunohistochemistry of Ki67 and p53; nevertheless, the logistic regression demonstrated that single the immunohistochemistry of p53 presented independent predictive value.

Conclusions: The determination of p53 presents predictive value of clinically useful progression in bledder tumors T1G3, so that its determination can constitute a essential factor in the strategies of treatment of these tumors.

Keywords: T1G3. Inmunohistoquemistry. P53. 


\section{INTRODUCCIÓN Y OBJETIVOS}

Los factores pronósticos clásicos no han demostrado ser suficientes para identificar aquellos tumores que van a progresar, antes de que ello ocurra. Por ello, se han analizado, en multitud de trabajos, nuevos factores pronósticos en el campo de la citogenética e inmunohistoquímica que pretenden caracterizar los diferentes potenciales biológicos del tumor, de manera que añadidos a los factores pronósticos clásicos, posibiliten el establecimiento de esquemas terapéuticos mas adecuados, con actitudes radicales aplicables antes de que la enfermedad progrese.

Nuestro objetivo ha sido determinar el valor predictivo de algunos de estos nuevos factores: la expresión inmunohistoquímica de algunas proteínas reguladoras del ciclo celular, como p53, p21 y Bcl-2 y la expresión del antígeno Ki-67 como marcador de proliferación celular. Una vez analizados individualmente, hemos seleccionado aquellos con valor predictivo independiente. La literatura al respecto de estos nuevos factores proporciona resultados a menudo enfrentados y además generalmente analiza grupos de tumores mas heterogéneos: tumores superficiales (Ta-T1), tumores T1 (G1-3), etc, siendo escasos los trabajos que particularizan en el grupo de tumores T1G3.

\section{MATERIAL Y MÉTODOS}

Hemos realizado un estudio retrospectivo sobre una serie inicial de 91 pacientes afectos de tumor vesical pT1G3, diagnosticados y tratados entre los años 1986 y 2003 en el Hospital Universitario La Fe de Valencia. Analizamos un total de 14 variables. Como factores pronósticos clásicos: edad, sexo, tabaquismo, número de tumores, tamaño tumoral, morfología (sésil versus pediculado), consistencia (sólido versus no sólido), localización, presencia o no de ureterohidronefrosis y carcinoma in situ asociado. Como factores inmunohistoquímicos determinamos el porcentaje de células inmunomarcadas y la intensidad de tinción para Ki-67 y las oncoproteinas p53, p21 y Bcl-2.

\section{Procesamiento de las muestras}

El procedimiento inmunohistoquímico se inició con el desparafinado de las muestras y poste- rior proceso de desenmascaramiento antigénico a partir de un tratamiento térmico en tampón citrato pH 6,0 (Buffer for antigen retrieval code. 2031, Dako, Dinamarca), utilizando como sistema de detección del antígeno el complejo EstreptavidaBiotina (labelled streptavidin-biotin LSAB, Dako, Dinamarca). Para el revelado de la técnica utilizamos el cromógeno 3.3-tetrahidrocloruro de diaminobencidina (DAB), obteniendo una reacción de color marrón. Posteriormente las muestras fueron contrastadas con hemotoxilina de Harris para la tinción en azul del resto de las estructuras de la muestra. Por último, las preparaciones se deshidrataron con baños consecutivos de alcohol 95\% y xilol y se montaron aplicando el medio de Eukitt (Lot.679; O.Kindler GMBH \& CO). En este punto llevamos a cabo dos tipos de controles: un control negativo, para comprobar la inespecificidad del anticuerpo secundario, y un control positivo, en el que utilizamos una muestra conocida que previamente habíamos seleccionado como positiva, asegurándonos de esta forma de que la técnica inmunohistoquímica se realizó correctamente.

La expresión nuclear de p53 se valoró utilizando el anticuerpo monoclonal DO-7 (N1581; Dako, Dinamarca), dilución 1:100. Las muestras fueron analizadas atendiendo al porcentaje de células tumorales que mostraban reacción positiva así como a la intensidad de tinción (negativa, intensidad baja, intensidad alta). Figura 1 y 2 .

La expresión de la proteína $\mathrm{p} 21^{\mathrm{WAF} 1}$ se estableció utilizando el anticuerpo monoclonal

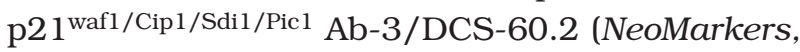
Fremont, USA), a una concentración de 4 microgramos/ml; en su expresión inmunohistoquímica se consideró también el porcentaje de células reactivas y la intensidad de tinción. Del mismo modo se procedió para el resto de inmunomarcadores; Ki-67: MIB-1 c126.1 (Biomeda, Foster City, CA, USA) dilución 1:50, y Bcl-2 antiboby C124 dilución 1:40 (Dako, Dinamarca).

El análisis de las laminillas se realizó por 2 observadores independientes y sin conocimiento de la evolución clínica de los tumores; en aquellos casos en que se obtuvieron resultados contradictorios se procedió a una reevaluación y se alcanzó un consenso.

Las pruebas estadísticas se llevaron a cabo con el programa SPSS para Windows versión 


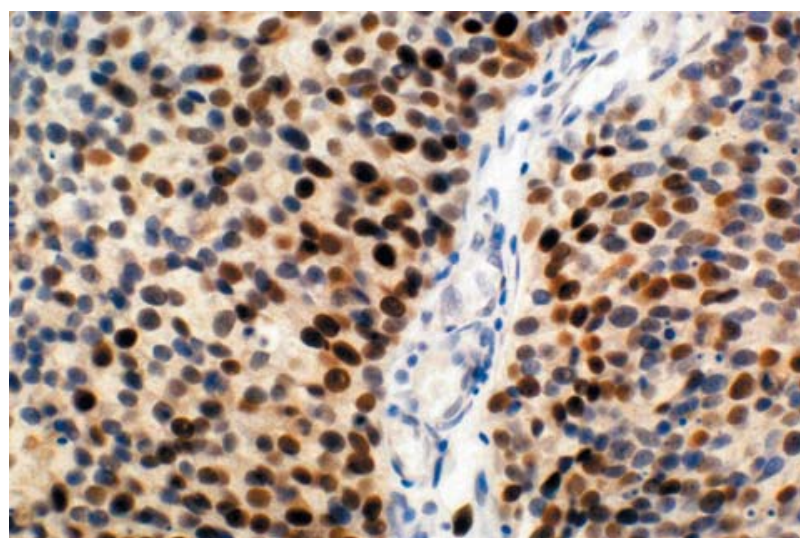

FIGURA 1. Inmunotinción para p53. Marcaje nuclear de más del $50 \%$ de células tumorales, con predominio de la intensidad de tinción alta. 400x

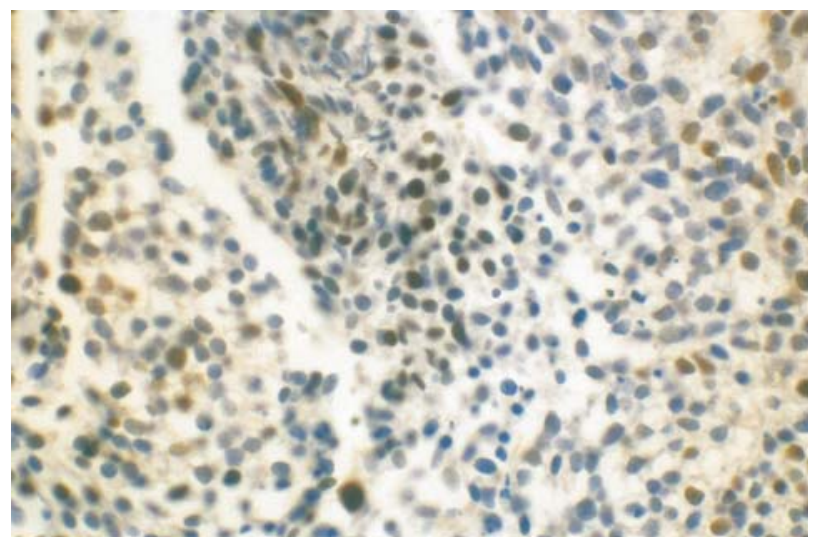

FIGURA 2. Inmunotinción para p53. Marcaje nuclear de menos del $50 \%$ de células tumorales, con predominio de la intensidad de tinción baja. 200x.

10.0, con una significación de 0,05 y las estimaciones con un intervalo de confianza del 95\%. Las funciones de supervivencia se establecieron por el método de Kaplan-Meier y el test de log-rank. Para determinar los factores pronósticos independientes utilizamos un modelo de regresión logistica.

\section{RESULTADOS}

De los 83 tumores, el 69\% (57) eran tumores T1G3 primarios y el 31\% de casos (26) procedían de un tumor superficial T1-Ta/G1-2. Todos fueron tratados con instilaciones intravesicales de BCG tras la RTU. El tiempo medio de seguimiento ha sido de 57,8 $\pm 28,2$ meses (rango: 13 124). Hubo recidiva en 60 pacientes. Un total de 31 han presentado infiltración del detrusor, existiendo actualmente 49 pacientes vivos sin enfermedad, 4 vivos con enfermedad, 13 exitus por causa no tumoral y 17 exitus de causa tumoral.

Los factores pronósticos clásicos con valor predictivo de progresión en el análisis univariante fueron el tamaño tumoral, la consistencia (sólida $v$ s friable), el numero de tumores, la morfología (sésil vs pediculada), la presencia de ureterohidronefrosis y el Cis asociado.

\section{Inmunohistoquímica de Ki 67}

La media de células inmunorreactivas fue de $43,5 \pm 27,2 \%$, mediana: $50 \%$, estableciendose el punto de corte en el 40\% (Ki67(-) si < 40\% células inmunomarcadas). Respecto a las tasas de progresión, de los 49 pacientes Ki67 (-) progresaron $14(29 \%)$ y de los $34 \mathrm{Ki} 67$ (+) progresaron 17 $(50 \%) \mathrm{p}<0,04$. Las curvas actuariales mostraron un menor tiempo libre de progresión en aquellos tumores con inmunotinción positiva para Ki-67, sin obtener diferencias significativas ni para el tiempo libre de recidiva ni para el tiempo de supervivencia. Figura 3.

En 66 de los 83 casos $(79,5 \%)$ las células inmunorreactivas presentaban una intensidad de tinción alta. La consideración de esta cualidad (aisladamente o en combinación con el porcentaje de células inmunorreactivas) en los diferentes análisis estadísticos, no logró incrementar el valor predictivo de considerar solo el contaje de células inmunorreactivas.

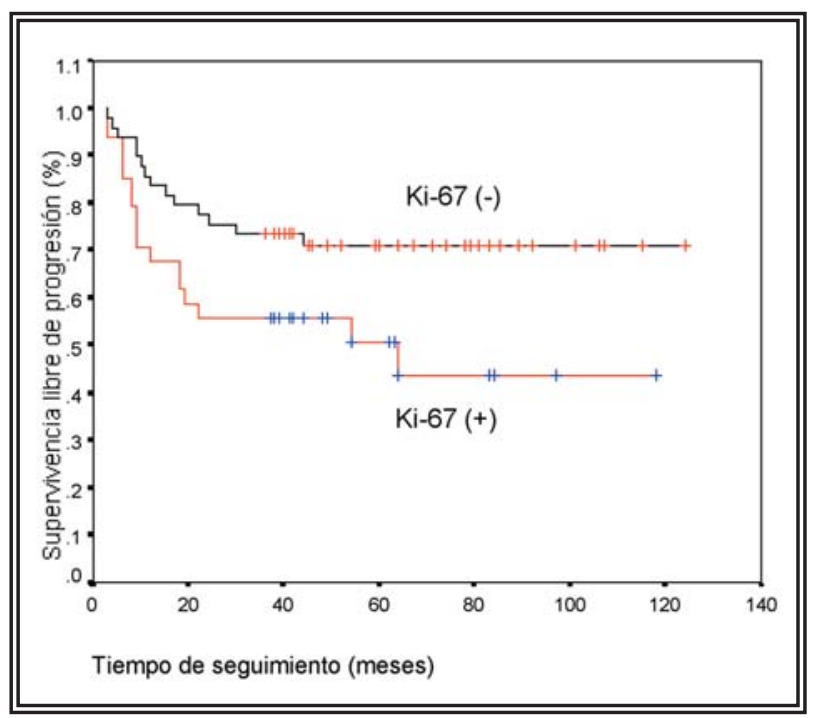

FIGURA 3. Probabilidad de progresión en función de la inmunotinción para Ki-67. 
Inmunohistoquímica de p53

La media de células marcadas por la presencia de la proteína p53 fué del 42,4 $\pm 25,7 \%$, mediana: 40\%. Al igual que para Ki-67, establecimos el punto de corte en el 40\%. Las tasas de progresión para cada grupo fueron: 12/51 (24\%) para p53 (-), y 19/32 (59\%) para p53 (+) $\mathrm{p}<0,002$. Además los pacientes con tumores p53 positivos presentaron un tiempo libre de progresión significativamente menor que aquellos con p53 negativo, sin apreciar diferencias en el tiempo libre de recidiva ni en el tiempo de supervivencia. Figura 4. Con este inmunomarcador la intensidad de tinción fue alta en el $50,6 \%$ de tumores y al igual que para $\mathrm{Ki}-67$, los diversos análisis estadísticos tampoco demostraron que la intensidad de tinción añadiera valor predictivo.

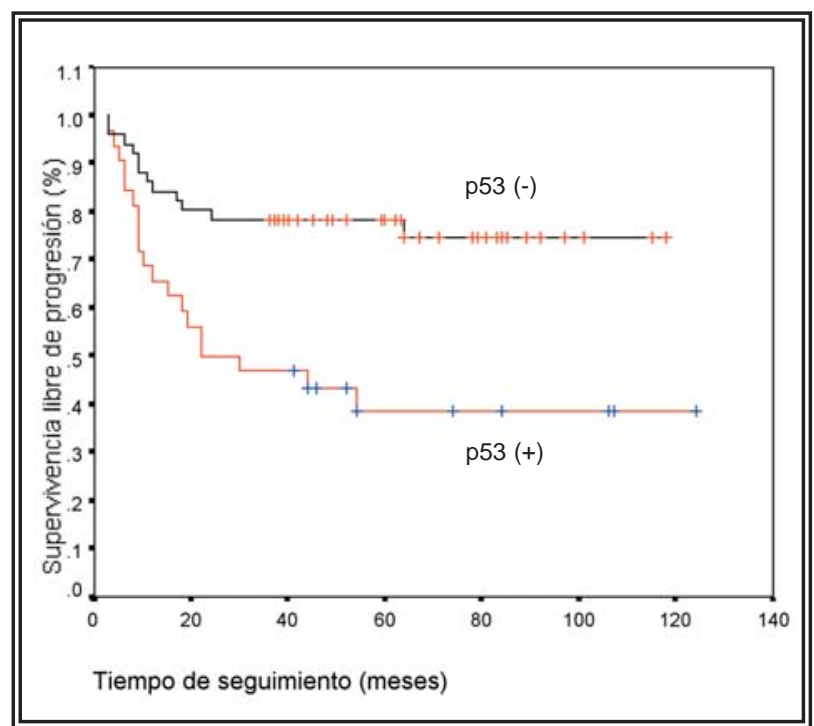

FIGURA 4. Probabilidad de progresión en función de la inmunotinción para p53.

\section{Inmunohistoquímica de p21}

La media de células con tinción positiva para este inmunomarcador fué de $22,4 \pm 20,8 \%$, con mediana del 20\%. Establecimos el punto de corte en el 20\%, no obteniendo significación estadística en ninguno de los análisis. Predominó la intensidad de tinción baja (38,6\% de casos), no hallando tampoco con esta característica, ningún valor estadísticamente significativo.

\section{Inmunohistoquímica de $\mathrm{Bcl}-2$}

Las células que presentaron inmunorreactividad con este marcador fueron escasas, con una media de $16,1 \pm 14 \%$ y mediana del 10\%. Tras establecer el punto de corte en el 10\%, no hallamos significación estadística ni con el tiempo libre de recidiva, tiempo libre de progresión o supervivencia. Predominó la intensidad de tinción alta (43\% de casos), constituyendo un factor significativo en cuanto al tiempo libre de recidiva, que fue menor cuanto mayor era la intensidad de tinción.

Por tanto, de todos estos factores inmunohistoquímicos, solo Ki-67 y p53 mostraron significación predictiva de progresión. La intensidad de tinción no demostró ninguna significación. El análisis multivariante, realizado con un modelo de regresión logística, que incluía los factores pronósticos clásicos y los factores inmunohistoquímicos, reveló como nueva variable pronóstica independiente la inmunohistoquímica de p53 (odds: 6,41) (Tabla 1).

\section{DISCUSIÓN}

Inmunohistoquímica de p53

Si analizamos la expresión inmunohistoquímica de p53 como factor pronóstico en el cáncer vesical existen muchas discrepancias y posturas enfrentadas. Muchas series no han demostrado

Tabla 1

Modelo de regresión logística. Factores pronósticos con valor predictivo independiente de progresión tumoral.

\begin{tabular}{lcccccc}
\hline & Coeficiente B Error Estándar & Wald $\mathrm{X}_{2}$ & $\mathrm{p}$ & Odds Ratio & $\begin{array}{c}\text { Intervalo de } \\
\text { Confianza (95\%) }\end{array}$ \\
\hline Constante & $-10,91$ & 2,55 & $18,67<0,001$ & & & \\
Multiplicidad & 1,96 & 0,76 & 7,47 & 0,0066 & 7,43 & $1,67-30,98$ \\
Tamaño (3 cm) & 0,75 & 0,63 & 1,37 & 0,2364 & 2,12 & $0,58-6,82$ \\
Cis & 0,37 & 0,62 & 0,29 & 0,5564 & 1,42 & $0,43-5,12$ \\
P53 & 1,95 & 0,61 & 9,11 & 0,0029 & 6,41 & $1,86-22,52$ \\
\hline
\end{tabular}


la utilidad de p53 como factor predictivo de progresión en tumores de alto riesgo ${ }^{1,2}$, ni de respuesta a $\mathrm{BCG}^{3}$; sin embargo, otros estudios si lo consideran útil, incluso le atribuyen valor pronóstico independiente de progresión ${ }^{4,5}$. Lee $\mathrm{E}$ y cols. ${ }^{6}$ en una serie de tumores de alto riesgo T1GII-III ponen de manifiesto, mediante análisis multivariante, que p53 es el único inmunomarcador clínicamente útil, frente a Ki-67, bcl-2, cathepsin-D, c-myc y C-erb-B2.

Pero, en relación al objetivo de este trabajo, que es obtener factores predictivos de progresión que permitan indicar la realización de una cistectomía precoz electiva, Steiner G y cols ${ }^{7}$, sobre una serie de tumores T1G3, consideran que la inmunohistoquímica de p53 no aporta información adicional a favor o en contra para dicha indicación; además el reciente estudio multicéntrico de Masters JR y cols. ${ }^{8}$ que agrupa un total de 502 tumores superficiales, muestra que, aunque p53 tiene valor pronostico independiente, la sensibilidad y especificidad de este inmunomarcador no es suficiente para establecer la indicación de cistectomía. En nuestra serie el análisis multivariante presenta a p53 como el único factor inmunohistoquímico con valor predictivo independiente, por lo que, aunque individualmente no permita indicar una cistectomía electiva, puede ayudar, asociado al resto de factores pronósticos.

Los motivos de discrepancia entre las distintas publicaciones son diversos, fundamentalmente en relación a la diversidad metodológica utilizada, tanto en el proceso de inmunotinción como en su posterior valoración ${ }^{9}$. Respecto a la primera consideración constituyen factores de perturbación los distintos medios en que está incluida la muestra (congelada, fijada en formalina, etc.), los métodos de recuperación del antígeno (calentamiento en microondas, ebullición, etc.) y el tipo de anticuerpo empleado (monoclonales o policlonales, de varias fuentes, etc.). Gardiner RA y cols. ponen de manifiesto la variabilidad de resultados en función de los diferentes anticuerpos y medios de inclusión utilizados $^{10}$. La valoración de la inmunoexpresión presenta dos inconvenientes, por una parte la selección de la laminilla(s) a analizar, con la propia heterogeneidad de la laminilla seleccio- nada, y por otra, el nivel de tinción que se considera como positivo. Se han utilizado diversos puntos de corte, estableciéndolo cada trabajo en función del que obtiene como óptimo. Los más utilizados en tumores superficiales han sido el 10 y $20 \%$. En nuestra serie, al tratarse de un grupo de alto grado, el nivel de expresión de p53 ha sido mayor, estableciéndose en el $40 \%$ de células inmunoreactivas.

También se deben tener en cuenta las diferencias Inter-observadores, e incluso intraobservador, en la interpretación del resultado inmunohistoquímico. En el estudio del National Center Institute de Maryland-USA ${ }^{11}$, se valoró la reproducibilidad de la medición de la expresión inmunohistoquímica de p53 en tumores vesicales, en 5 instituciones diferentes. Dicha reproducibilidad fue buena únicamente en los casos de baja y alta proporción de células inmunoreactivas, existiendo reducido nivel de concordancia en la gama intermedia.

En la amplia revisión de la literatura que realizan Schmitz-Drager BJ y cols. ${ }^{12}$ sobre 138 publicaciones que analizan el valor predictivo de p53, obtienen que en el $67 \%$ de ellas se comunica una correlación positiva con la evolución de la enfermedad. Sin embargo, no puede realizar un meta-análisis del conjunto de series por la heterogeneidad de tipos tumorales, anticuerpos utilizados, puntos de corte, métodos de análisis de la muestra, etc. El mismo autor considera que actualmente se hace necesaria la determinación y evaluación de p53 bajo el establecimiento de unos protocolos estandarizados, así como la realización de un ensayo multicéntrico prospectivo que proporcione respuestas definitivas.

\section{Inmunohistoquímica de Ki-67}

La expresión inmunohistoquímica nuclear del antígeno Ki-67, cuyo anticuerpo mas específico es el MIB-1, se ha relacionado tanto con el estadio como con el grado tumoral ${ }^{13,14}$. Diversos son los estudios que han demostrado su valor predictivo en tumores vesicales superficiales, incluso como factor pronóstico independiente de progresión ${ }^{15-18}$. Rodríguez Alonso y cols. ${ }^{19}$ consideran que el estado de Ki67 presenta valor pronóstico independiente de progresión, de 
manera que cuanto mayor es el porcentaje de expresión de Ki67 mayor es el riesgo. Pero también los hay que no le dan valor predictivo independiente ${ }^{3,20}$. Sobre el grupo específico de tumores T1G3 Wolf HK y cols. ${ }^{21}$ no encuentran ningún valor y Lebret $\mathrm{T}$ y cols. ${ }^{22}$ aunque consideran que este marcador puede predecir los pacientes respondedores a BCG, no predice los no respondedores y por tanto no es capaz de seleccionar los candidatos a cistectomía.

En nuestra serie, aunque el análisis univariante demostró que aquellos pacientes considerados como Ki-67(+) presentaban un tiempo libre de progresión significativamente menor que aquellos considerados Ki-67 (-), el analisis multivariante no lo señaló como factor predictivo independiente de progresión.

Los resultados contradictorios de las distintas publicaciones ponen de manifiesto que el empleo de este inmunomarcador en la práctica clínica es limitado, siendo quizás más un factor pronóstico de recidiva que de progresión. Considerando el análisis multivariable, la inmunohistoquímica de Ki-67 no es un marcador pronóstico independiente establecido.

\section{Inmunohistoquímica de $p 21$}

La proteína p21 se expresa a partir del gen $p 21$, activado por la proteína p53; por ello las alteraciones a nivel del gen p53 pueden alterar la expresión de p21. Pero algunos autores ${ }^{23}$ han demostrado también su regulación por otras vias independientes de p53. En nuestra serie no hemos observado correlación entre los niveles de ambos inmunomarcadores.

En cuanto a la importancia pronóstica de la expresión de p2 1 existe controversia. La mayoría de trabajos no la señalan como un factor predictivo independiente en tumores superficiales $^{24-28}$, incluidos aquellos que consideran específicamente los tumores de alto riesgo y T1G3 ${ }^{3,21}$.

Algunos trabajos asocian la expresión de p2 1 con la de p53 en un intento de mejorar la capacidad pronóstica de ambos: Stein JP y cols. ${ }^{29}$ analizaron la expresión de p21 y p53 en una serie de 242 pacientes con tumores superficiales e invasivos; además de observar que la expresión de p21 mostraba, en el análisis mul- tivariante, un valor predictivo de recidiva y de supervivencia, encontraron un efecto pronóstico cooperativo con p53, de manera que los pacientes con sobreexpresión de p53 y con p21 negativo, eran los que mostraban peor supervivencia, manteniendo longevidades similares aquellos que conservaban la expresión de p21 independientemente del estado de p53.

En nuestro trabajo no se ha observado que p21 posea un valor pronóstico significativo, ni por sí mismo ni en cooperación con p53.

\section{Inmunohistoquímica de $\mathrm{Bcl}-2$}

La proteína Bcl-2 es un potente inhibidor de la apoptosis, por lo que su sobre-expresión se ha asociado a un déficit apoptótico y a la consiguiente proliferación de las células tumorales. $\mathrm{Su}$ valor como factor predictivo en el cáncer vesical está todavía también por determinar. Los estudios recientes mayoritariamente consideran que la expresión inmunohistoquímica de esta proteína no presenta un valor significativo ni en el análisis univariante ni en el multivariante $^{1,}$ 30-34. En tumores pT1G3, Wolf HK y cols. ${ }^{21}$ piensan que la positividad de Bcl-2 identifica tumores más agresivos, pero Plastiras D y $\operatorname{cols}^{34}$ en una serie de tumores de alto riesgo no dan ningún valor pronóstico a esta proteína. En nuestra serie tampoco encontramos valor predictivo significativo.

\section{CONCLUSIONES}

De entre los nuevos factores predictivos propuestos, solo presentan información clínicamente útil, la inmunohistoquímica de p53. Este marcador puede ser establecido sin requerir especiales condiciones de laboratorio. Sin embargo la disparidad de resultados publicados en relación a p53 hace que se deba establecer una técnica estandarizada y universal que definitivamente establezca su papel en la predicción de la progresión tumoral. A tenor de nuestros resultados consideramos que la determinación inmunohistoquímica de la proteína p53 como factor pronóstico es fundamental para el establecimiento de las estrategias de tratamiento de los pacientes con tumores vesicales pT1G3. 


\section{REFERENCIAS}

1. Stavropoulos NE, Filiadis I, Ioachim E et al. Prognostic significance of p53, bcl-2 and Ki-67 in high risk superficial bladder cancer. Anticancer Res 2002; 22:3759-3764.

2. Gil P, Allepuz C, Blas M, et al. Significance of protein p53 overexpression in the clinical course of high-risk superficial bladder cancer. Urol Int 2003;70:172-177.

3. Zlotta AR, Noel JC, Fayt I, et al. Correlation and prognostic significance of p53, p21waf1/cip1 and Ki-67 expression in patients with superficial bladder tumors treated with bacillus calmette-guerin intravesical therapy. J Urol 1999;161:792-798.

4. Malmstrom PU, Wijkstrom H, Lundholm C, Wester K, Busch C, Norlen BJ. 5-year followup of a randomized prospective study comparing mitomycin $\mathrm{C}$ and bacillus Calmette-Guerin in patients with superficial bladder carcinoma. Swedish-Norwegian Bladder Cancer Study Group. J Urol 1999;161: 1124-1127.

5. Lacombe L, Dalbagni G, Zhang ZF, et al. Overexpression of p53 protein in a high-risk population of patients with superficial bladder cancer before and after bacillus calmette-guerin therapy: correlation to clinical outcome. J Clin Oncol 1996;14:2646-2652.

6. Lee E, Park I, Lee C. Prognostic markers of intravesical bacillus calmette-guerin therapy for multiple, high-grade, stage T1 bladder cancers. Int J Urol 1997;4:552-556.

7. Steiner G, Bierhoff E, Schmidt D, Leissner J, Wolf HK, Albers P. p53 immunoreactivity in biopsy specimens of T1G3 transitional cell carcinoma of the bladder-a helpful parameter in guiding the decision for or against cystectomy?. Eur J Cancer 2000;36:610-614.

8. Masters JR, Vani UD, Grigor KM, et al. MRC Superficial Bladder Cancer Group Mitomycin-C Trial Collaborators. Can p53 staining be used to identify patients with aggressive superficial bladder cancer?. J Pathol 2003;200:74-81.

9. Helpap B, Schmitz-Drager BJ, Hamilton PW, et al. Molecular pathology of non-invasive urothelial carcinomas (part I). Virchows Arch 2003;442:309-316.

10. Gardiner RA, Walsh MD, Allen V, et al. Immunohistological expression of p53 in primary pT1 transitional cell bladder cancer in relation to tumour progression. $\mathrm{Br} \mathrm{J}$ Urol 1994;73:526-532.

11. McShane LM, Aamodt R, Cordon-Cardo C, et al. Reproducibility of p53 immunohistochemistry in bladder tumors. National Cancer Institute, Bladder Tumor Marker Network. Clin Cancer Res 2000;6:1854-1864.

12. Schmitz-Drager BJ, Goebell PJ, Ebert T, Fradet Y. p53 immunohistochemistry as a prognostic marker in bladder cancer. Playground for urology scientists?. Eur Urol 2000;38:691-699.

13. Liukkonen TJ, Lipponen PK, Helle M, Haapasalo HK, Nordling S, Rajala P. Expression of MIB-1, mitotic index and s-phase fraction as indicators of cell proliferation in superficial bladder cancer. Finnbladder Group. Urol Res 1996;24:61-66.

14. Pfister C, Lacombe L, Vezina MC. Prognostic value of the proliferative index determined by Ki-67 immunostaining in superficial bladder tumors. Hum Pathol 1999;30:13501355.
15. Liukkonen T, Rajala P, Raitanen M, Rintala E, Kaasinen E, Lipponen P. Prognostic value of MIB-1 score, p53, EGFr, mitotic index and papillary status in primary superficial (Stage pTa/T1) bladder cancer: a prospective comparative study. The Finnbladder Group. Eur Urol 1999;36:393400.

16. Popov Z, Hoznek A, Colombel M, et al. The prognostic value of p53 nuclear overexpression and MIB-1 as a proliferative marker in transitional cell carcinoma of the bladder. Cancer 1997; 80:1472-1481.

17. Blanchet P, Droupy S, Eschwege P, et al. Prospective evaluation of Ki-67 labeling in predicting the recurrence and progression of superficial bladder transitional cell carcinoma. Eur Urol 2001;40:169-175.

18. Santos L, Amaro T, Costa C, et al. Ki-67 index enhances the prognostic accuracy of the urothelial superficial bladder carcinoma risk group classification. Int $J$ Cancer 2003; 105:267-272.

19. Rodríguez-Alonso A, Pita-Fernandez S, Gonzalez-Carrero J, Nogueira-March JL. Multivariate analysis of recurrence and progression in stage $\mathrm{T} 1$ transitional cell bladder carcinoma. Prognostic Value of p53 and Ki67. Actas Urol Esp 2003;27:132-141.

20. Nakopoulou L, Vourlakou C, Zervas A, Tzonou A, Gakiopoulou H, Dimopoulos MA. The prevalence of bcl-2, p53 and Ki-67 immunoreactivity in transitional cell bladder carcinomas and their clinicopathologic correlates. Human Pathol 1998;29:146-154.

21. Wolf HK, Stober C, Hohenfellner R, Leissner J. Prognostic value of p53, p21/waf1, bcl-2, bax, bak and Ki-67 immunoreactivity in pT1G3 urothelial bladder carcinomas. Tumour Biol 2001;22: 328-336.

22. Lebret T, Becette V, Herve JM, et al. Prognostic value of Mib-1 antibody labeling index to predict response to bacillus calmette-guerin therapy in a high-risk selected population of patients with stage T1 grade G3 bladder cancer. Eur Urol 2000;37:654-659.

23. Michieli P, Chedid M, Lin D, Pierce JH, Mercer WE, Givol D. Induction of waf1/cip1 by p53 independent pathway. Cancer Res 1994;54:3391-3395.

24. Leissner J, Hohenfellner R, Thuroff JW, Koppen C, Wolf HK. Prognostic significance of histopathological grading and immunoreactivity for p53 and p21/WAF1 in grade 2 pTa transitional cell carcinoma of the urinary bladder. Eur Urol 2001;39:38- 445.

25. Chow NH, Tzai TS, Cheng HL, Liu HS, Chan SH, Tong YC. The clinical value of $\mathrm{p} 21$ waf1/cip1 expression in superficial bladder cancer. Anticancer Res 2000;20:1173-1176.

26. Liukkonen T, Lipponen P, Raitanen M, et al. Evaluation of p21waf1/cip 1 and cyclin d1 expression in the progression of superficial bladder cancer. Finbladder group. Urol Res 2000; 28:285-292.

27. Lipponen P, Aaltomaa S, Eskelinen M, Ala-Opas M, Kosma VM. Expression of p21 (WAF1/CIP1) protein in transitional cell bladder tumours and its prognostic value. Eur Urol 1998;34:237-243.

28. Pfister C, Moore L, Allard P, et al. Predictive value of cell cycle markers p53, MDM2, p21, and Ki-67 in superficial bladder tumor recurrence. Clin Cancer Res 1999;5:40794084. 
29. Stein JP, Ginsberg DA, Grossfeld GD, et al. Effect of p21waf1/cip1 expression on tumor progression in bladder cancer. J Natl Cancer Inst 1998;90:1072-1079.

30. Hussain SA, Ganesan R, Hiller L, et al. Proapoptotic genes bax and cd 401 are predictors of survival in transitional cell carcinoma of the bladder. Br J Cancer 2003;88:586-592.

31. Yan Y, Andriole GL, Humphrey PA, Kibel AS. Patterns of multiple recurrences of superficial $(\mathrm{Ta} / \mathrm{T} 1)$ transitional cell carcinoma of bladder and effects of clinicopathologic and biochemical factors. Cancer 2002;95:1239-1246.

32. Pich A, Chiusa L, Formiconi A, et al. Proliferative activity is the most significant predictor of recurrence in noninvasive papillary urothelial neoplasms of low malignant potential and grade 1 papillary carcinomas of the bladder. Cancer 2002;95:784-790.

33. Wu TT, Chen JH, Lee YH, Huang JK. The role of bcl-2, p53, and ki-67 index in predicting tumor recurrence for low grade superficial transitional cell bladder carcinoma. J Urol 2000;163:758-760.

34. Plastiras D, Moutzouris G, Barbatis C, Presvelos V, Petrakos M, Theodorou C. Can p53 nuclear over-expression, bcl-2 accumulation and pcna status be of prognostic significance in high-risk superficial and invasive bladder tumours?. Eur J Surg Oncol 1999;25:61-65.

Dr. J.A. Queipo Zaragozá

Calle Santa Teresa № $4-\mathrm{P}^{\mathrm{a}} 8$

46530 Puzol (Valencia)

E-mail: queipo@pulso.com

(Trabajo recibido el 21 noviembre de 2004) 